\title{
The Talking Quilt - Augmenting Domestic Objects for Communal Meaning-Making
}

\author{
Sara Heitlinger \\ Queen Mary University of London \\ Media and Arts Technology \\ Mile End Road \\ London E1 4NS \\ United Kingdom \\ sara@eecs.qmul.ac.uk
}

\author{
Nick Bryan-Kinns \\ Queen Mary University of London \\ C4DM \\ Mile End Road \\ London E1 4NS \\ United Kingdom \\ nick.bryan-kinns@eecs.qmul.ac.uk
}

\author{
Tony Stockman \\ Queen Mary University of London \\ IMC \\ Mile End Road \\ London E1 4NS \\ United Kingdom \\ tony.stockman@eecs.qmul.ac.uk
}

\author{
Orla O'Flanagan \\ ActiveArt \\ London \\ United Kingdom \\ orla@active-art.org
}

\author{
Tarot Couzyn \\ ActiveArt \\ London \\ United Kingdom \\ tarot@active-art.org
}

\section{INTRODUCTION}

The Talking Quilt is a traditional textiles quilt that has been augmented with digital technology to allow for enjoyable and reflective engagement. Produced by over 80 staff, volunteers and visitors to Spitalfields City Farm in East London, the quilt presents a snapshot of the farm at this point in time. Measuring $2 \times 1$ metres, it consists of over a thousand hexagonal fabric patches, many of which have been hand-printed and painted by participants, and with specific significance to their makers' lives. New technology in the form of RFID (radio frequency identification) buttons that trigger playback of oral history interviews is used to bring the everyday domestic object to life, to make it contemporary and augment the experience of interacting with it without changing its qualities as a visual, tactile, and hand-made object. Rather than aiming for a solely playful interaction, the research looked at how socially meaningful themes integral to the quilt - i.e. food, food growing and community - could be communicated through the interaction.

\section{DESIGN RATIONALE}

The design challenge was how to merge the physical, social and technological aspects of the quilt in complementary ways. We drew inspiration from the aims of Ludic design (Gaver et al., 2006) and Slow Technology (Hallnäs \& Redström, 2001) to allow for a reflective and personally-motivated form of engagement. It was important for the collective labour to be evident in the technological intervention and for a communal reception to be possible. In addition, the quilt was designed to be viewed, scanned and listened to simultaneously in a holistic way, with 150 audio tracks and over 50 different voices.

\section{OBSERVATIONS}

Audience's responses from 3 public exhibitions suggest that the quilt allows for a reflective interaction: "It was nice, it slowed you down...It is playful to look at. But it's much nicer to not be playful in using it, to work through the stories, hear them and take time with them.' The collective nature of the quilt was mentioned: "I love the idea of the voices, singly, but also that they can be combined in this waterfall of speech that washes over you." Audiences displayed different levels of engagement with the content. We divide this into: a surface engagement - where users thought the quilt was a nice object but didn't engage with the content; an associative engagement - the general themes were understood and associations triggered; and a deep engagement - specific reference to stories heard were made, e.g., "I remember one story about someone being made of fish and rice because they were Bengali, and I'm half Bengali so I can relate to that one."

\section{REFERENCES}

Gaver, W., Bowers, J., Boucher, A., Law, A., Pennington, S., and Villar, N. (2006) The History Tablecloth: Illuminating domestic activity. DIS, New York, USA, pp. 199-208. 
The Talking Quilt - Augmenting Domestic Objects for Communal Meaning-Making

Sara Heitlinger, Nick Bryan-Kinns, Tony Stockman, Orla O'Flanagan \& Tarot Couzyn

Hallnäs, L. and Redström, J. (2001) Slow Technology - Designing for Reflection. Personal Ubiquitous Computing, 5(3):201-212.

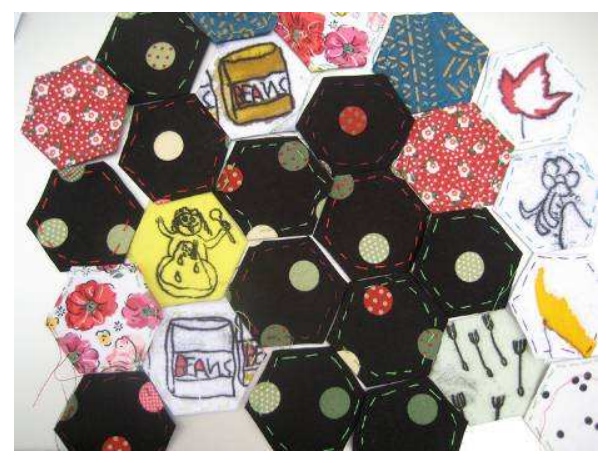

Figure 1: Some of the patches, many of which were hand-painted and printed, and with personal meaning for their makers' lives.

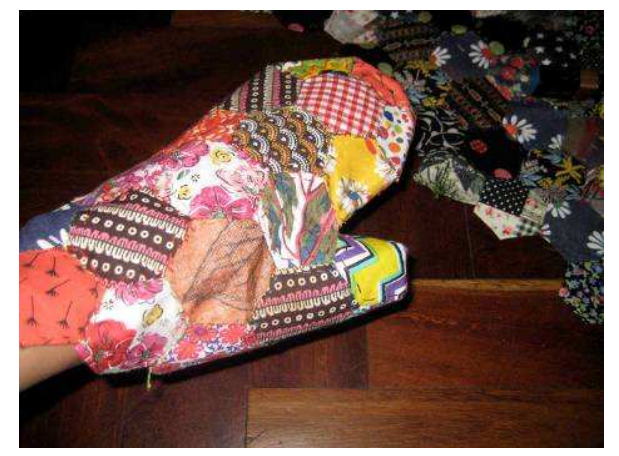

Figure 2: RFID-enabled oven glove used to scan the quilt and trigger audio playback

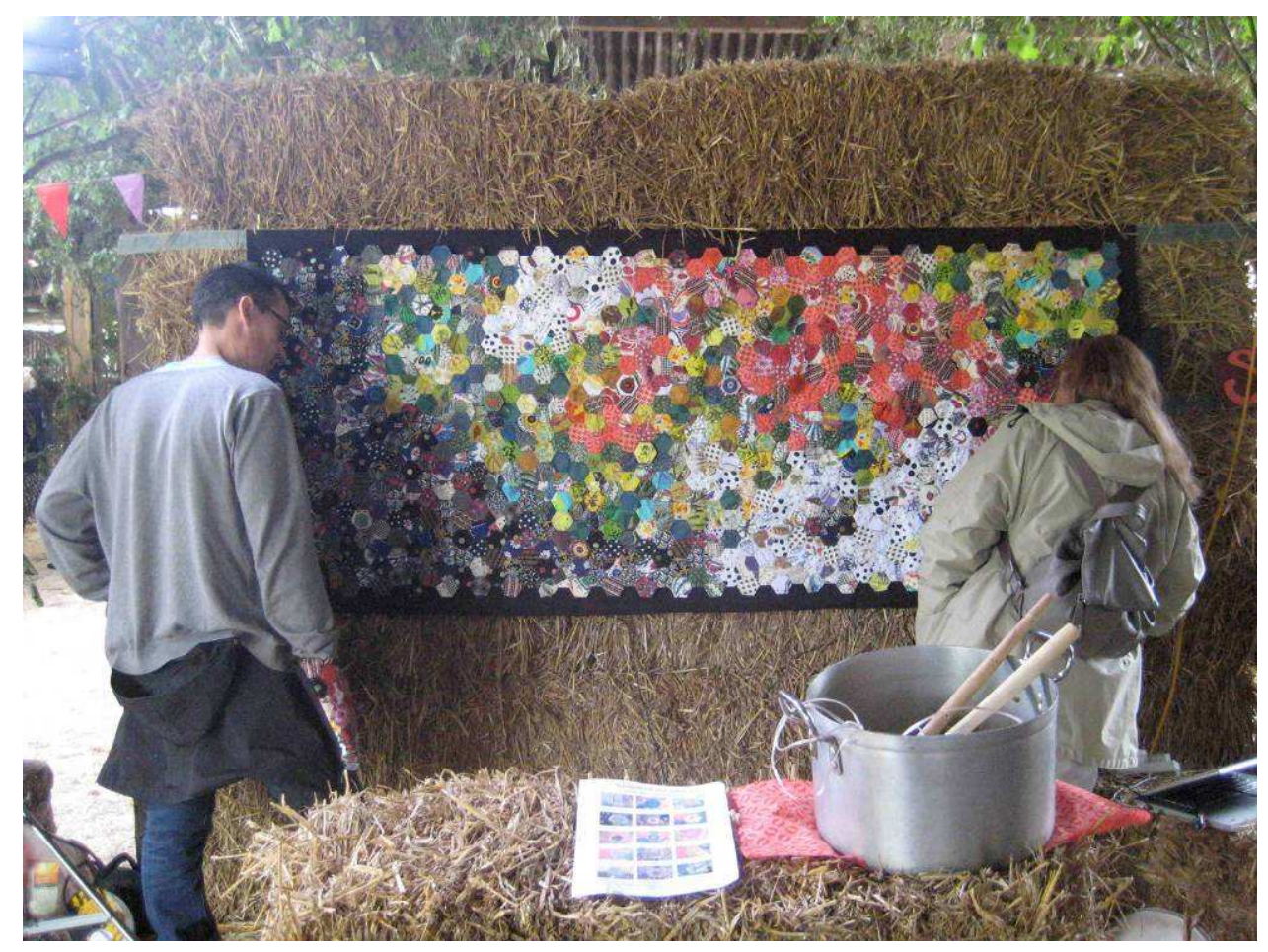

Figure 3: Audience members listening to the quilt 